OPEN

SUBJECT AREAS:

QUANTUM

INFORMATION

QUBITS

Received

15 October 2013

Accepted

27 November 2013

Published

7 January 2014

Correspondence and requests for materials should be addressed to

X.-S.M. (Xiaosong.

Ma@univie.ac.at)

\title{
Towards photonic quantum simulation of ground states of frustrated Heisenberg
} spin systems

Xiao-song Ma ${ }^{1,2,3}$, Borivoje Dakić4,5,6, Sebastian Kropatschek1, William Naylor' ${ }^{1}$ Yang-hao Chan 7,8 , Zhe-xuan Gong ${ }^{7,8}$, Lu-ming Duan ${ }^{7,8}$, Anton Zeilinger ${ }^{1,2}$ \& Philip Walther ${ }^{4}$

'Institute for Quantum Optics and Quantum Information (IQOQI), Austrian Academy of Sciences, Boltzmanngasse 3, A-1090 Vienna, Austria, ${ }^{2}$ Vienna Center for Quantum Science and Technology, Faculty of Physics, University of Vienna, Boltzmanngasse 5, A-1090 Vienna, Austria, ${ }^{3}$ Department of Electrical Engineering, Yale University, 15 Prospect Street, New Haven, CT 06520, USA, ${ }^{4}$ Faculty of Physics, University of Vienna, Boltzmanngasse 5, A-1090 Vienna, Austria, ${ }^{5}$ Centre for Quantum Technologies, National University of Singapore, 3 Science Drive 2, Singapore 1 17543, ${ }^{\circ}$ Clarendon Laboratory, University of Oxford, Parks Road, Oxford OX1 3PU, UK, ${ }^{7}$ Department of Physics and MCTP, University of Michigan, Ann Arbor, MI 48109, USA, ${ }^{8}$ Center for Quantum Information, IIIS, Tsinghua University, Beijing, China.

Photonic quantum simulators are promising candidates for providing insight into other small- to medium-sized quantum systems. Recent experiments have shown that photonic quantum systems have the advantage to exploit quantum interference for the quantum simulation of the ground state of Heisenberg spin systems. Here we experimentally characterize this quantum interference at a tuneable beam splitter and further investigate the measurement-induced interactions of a simulated four-spin system by comparing the entanglement dynamics using pairwise concurrence. We also study theoretically a four-site square lattice with next-nearest neighbor interactions and a six-site checkerboard lattice, which might be in reach of current technology.

M ore than a quarter of a century ago, Richard Feynman ${ }^{1,2}$ envisioned that a well-controlled quantum mechanical system can be used for the efficient simulation of other quantum systems and thus is capable of calculating properties that are unfeasible for classical computers. Quantum simulation promises potential returns in understanding detailed quantum phenomenon of inaccessible quantum systems ${ }^{3}$, from molecular structure to the behavior of high-temperature superconductors. Moreover, quantum simulations are conjectured to be less demanding than quantum computations by being less stringent on explicit gate operations or error correction ${ }^{4,5}$. Due to these reasons, quantum simulation has led to many theoretical proposals $^{6-9}$. Recently, various quantum simulators based on different physical platform are being constructed, such as atoms in optical lattice ${ }^{10-15}$, trapped ions ${ }^{16-21}$, nuclear magnetic resonance $e^{22,23}$, superconducting circuits ${ }^{24}$, as well as single photons ${ }^{25-33}$. For recent reviews, see ref. 34-37.

Motivated by the seminal work of Knill, Laflamme and Milburn ${ }^{38}$ photons are proven to be a suitable system for efficient quantum computing and quantum simulation. Precise single-photon manipulations and tunable measurement-induced two-photon interactions are the essential ingredients for photonic analog quantum simulation and have been demonstrated. In addition to the high-level quantum control, such photonic quantum simulators can produce exotic entangled states which are important for understanding the many-body dynamics in quantum chemistry and solid-state physics ${ }^{8,9,31}$. Note that the recently proposed universal quantum computation based on coherent photon conversion provides an alternative avenue for achieving photon-photon interaction ${ }^{39}$.

In ref. 31, a spin tetramer, the simplest example of the Heisenberg-interacting spin system, has been investigated. In this work we will not only present the detailed study on the tunable measurement-induced photonphoton interaction, but also further investigate more complicated Heisenberg models for the four-site square lattice with nearest and next-nearest couplings, and the six-site checkerboard lattice.

Analog quantum simulations with photons and linear optics

In the case of the simulation of spin-1/2 particles the photon's polarization is ideally suited as horizontallypolarized states $|H\rangle$ and vertically-polarized states $|V\rangle$ represent for example spin-up and spin-down states. Moreover, the ability to prepare symmetric polarization-entangled states $|\Phi\rangle_{1,2}^{ \pm}=\frac{1}{\sqrt{2}}\left(|H\rangle_{1}|H\rangle_{2} \pm|V\rangle_{1}|V\rangle_{2}\right)$, 
$|\Psi\rangle_{1,2}^{+}=\frac{1}{\sqrt{2}}\left(|H\rangle_{1}|V\rangle_{2}+|V\rangle_{1}|H\rangle_{2}\right)$ and the anti-symmetric state $\left|\Psi^{-}\right\rangle_{1,2}=\frac{1}{\sqrt{2}}\left(|H\rangle_{1}|V\rangle_{2}-|V\rangle_{1}|H\rangle_{2}\right)$ enables the establishment of states with bosonic and fermionic character ${ }^{30,31,40}$. The latter shares the same quantum correlations as Heisenberg-interacting spins or so-called valence bond states ${ }^{41}$.

The theoretical investigation of strongly-correlated spin systems has led to few exact theorems which in some cases are of importance for the quantum simulation of chemical and physical models. In the particular case of a nearest-neighbor antiferromagnetic Heisenberginteracting spin system it was shown by Marshall ${ }^{42}$ that the ground state for $\mathrm{N}$ spins on a bipartite lattice has total spin zero. This theorem and its extension ${ }^{43}$ lead to the fact that the ground state must be built as a linear superposition of singlet spin states or valence bonds. This constraint, that forces the ground state's total spin to be zero, gives rise to various valence-bond configurations that are either localized or fluctuating as superposition of different singlet partitionings. Localized configurations are typically referred to valence bond solids and delocalized valence-bond states correspond to frustrated quantum spin liquids or resonating valence-bond states ${ }^{44,45}$. Recently, the photonic quantum simulation of a four spin-1/2 square lattice as an archetype system ${ }^{31}$ showed that quantum monogamy ${ }^{46,47}$ plays an important role in frustrated Heisenberg spin systems.

The experimental setup for studying variable measurementinduced interactions is shown in Fig. 1. Our pump source is a mode-locked Ti:sapphire femto-second laser with a pulse duration of $140 \mathrm{fs}$ and a repetition rate of $80 \mathrm{MHz}$. The central wavelength of the pump is at $808 \mathrm{~nm}$. Then we use a $\beta$-barium borate crystal (BBO0) to up-convert the pump pulses to ultraviolet (UV) pulses via second harmonic generation. The up-converted UV pulses' central wavelength is $404 \mathrm{~nm}$ with a pump power of $700 \mathrm{~mW}$. Then we clean the UV pulses with several dichroic mirrors (DM). Photons 1 and 2 are generated from a BBO crystal (BBO1) via spontaneous parametric down conversion (SPDC) in a non-collinear type-II phase matching configuration and in an polarization-entangled state after walk-off compensation ${ }^{48}$. Photons 3 and 4 are generated from $\mathrm{BBO} 2$ in a collinear type-II phase matching configuration and are separated by a polarizing beam splitter (PBS). We guide photons 1 and 3 to a tunable directional coupler (TDC) and then detect them by avalanche photodiodes (APD), which together enable the tunable measurement-induced photon-photon interactions to happen. The relative temporal delay between the photons is adjusted with a motorized translating stage mounted on the fiber coupler of photon 1. Fiber polarization controllers (PC) are employed to eliminate the polarization distinguishability of the two interfering photons.

Bunching due to the two-photon Hong-Ou-Mandel (HOM) interference ${ }^{49}$ and the corresponding anti-bunching effect ${ }^{40}$ are crucial for many quantum information processing protocols, especially for photonic quantum computation experiments (C-phase gat $\mathrm{e}^{50,51}$, entanglement swapping ${ }^{52,53}$, etc.), as well as for our photonic quantum simulation $^{31}$. The bosonic nature of the photons shows up when the input states are superimposed at the TDC such that a detection, even in principle, cannot distinguish either of them. This leads to a superposition of double occupations on both outgoing modes and thus suppression of the coincidence detections, where one photon is detected in each output mode. The visibility of this HOM dip is one when the TDC is set to have equal splitting of transmitted and reflected photons similar to a 50/50 beam splitter. As soon as the two input photons can be partially distinguished by

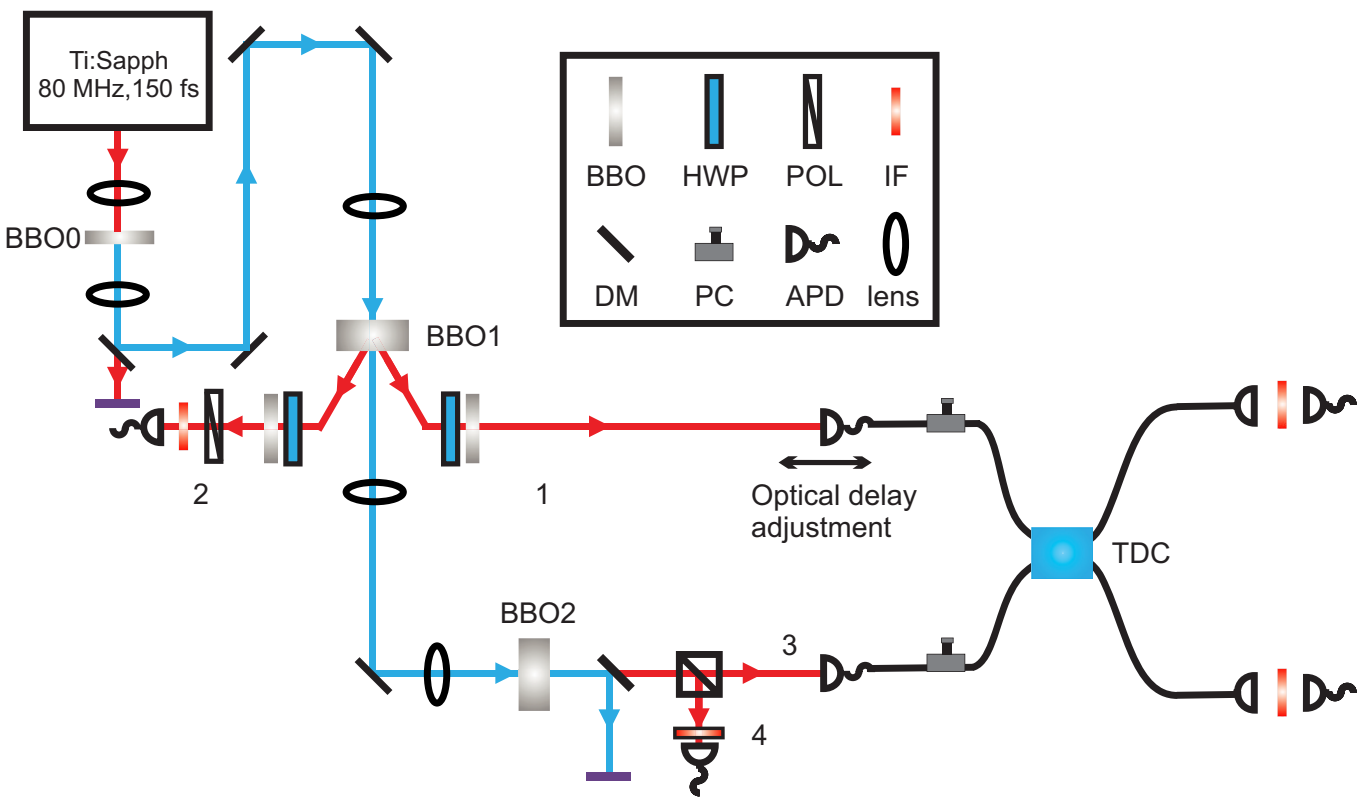

Figure 1 Experimental setup for testing variable measurement-induced interaction between two independent photons. Ultraviolet (UV) femtosecond laser pulses are up-converted from a mode-locked Ti:sapphire laser. The laser pulses are up converted (BBO0) and the obtained temporal duration and repetition rate is $140 \mathrm{fs}$ and $80 \mathrm{MHz}$, respectively. The UV pulses and the remaining fundamental pulses are separated with several dichroic mirrors (DM). Lenses are used to focus and collimate the UV pulses in order to achieve good up and down conversion efficiencies. One pair of the polarization entangled photons (photons 1 and 2 ) is generated from a $\beta$-barium borate crystal (BBO1) via spontaneous parametric down conversion (SPDC) in a noncollinear type-II phase matching configuration. Two half-wave plates (HWP) and compensating BBO crystals are used to counter walk-off effects in the down-conversion crystal. Another pair of correlated photons (photons 3 and 4) is generated from BBO2 in a collinear type-II phase matching configuration and are separated by a polarizing beam splitter (PBS). Single-mode fibers and interference filters (IF) are used to clean their spatial and spectral modes, respectively. We vary the path length difference between two photons with a motorized translation stage mounted on the fiber coupling stage of photon 1. Fiber polarization controllers (PC) are employed to eliminate the polarization distinguishability of the two interfering photons. The tunable measurement-induced interaction among photons is achieved by a tunable directional coupler (TDC), followed by a projective measurement of one photon in each of the four output modes. 
unbalancing the splitting ratio the visibility decreases. The dependence of the ideal visibility $\left(V_{\text {ideal }}\right)$ upon the reflectivity of TDC $(\eta)$ is the following:

$$
V_{\text {ideal }}=\frac{2 \eta(1-\eta)}{1-2 \eta+2 \eta^{2}} .
$$

In Fig. 2 this reflectivity dependent ideal visibility is plotted in the black solid curve. Experimental imperfections due to high-order emissions from SPDC and group velocity mismatch reduce $V_{\text {ideal }}$. The measured visibilities (black squares) are also shown as well as the corresponding fit (dashed red curve).

Pair-wise entanglement dynamics. The main advantage of the precise quantum control of individual particles is that inter-particle entanglement dynamics can be investigated. By using a similar experimental configuration as in Ref. 31, we study the entanglement distributions among different particles with respect to the effective interaction strength that was tuned by the TDC. For the quantification of the bipartite entanglement in our system, we use the measure of concurrence ${ }^{54}$, which, for a given state $\rho$, is $C(\rho) \equiv \max \left\{0, \sqrt{\lambda_{1}}-\sqrt{\lambda_{2}}-\sqrt{\lambda_{3}}-\sqrt{\lambda_{4}}\right\}$, where $\lambda_{i}$ are the eigenvalues of the matrix $\rho \Sigma \rho^{T} \Sigma$ in non-increasing order by magnitude with $\Sigma=\sigma_{Y} \otimes \sigma_{Y}$, where $\sigma_{Y}=-i|0\rangle\langle 1|+i| 1\rangle\langle 0|$. While the previous quantum simulation characterized the pair-wise energy dynamics of Heisenberg interactions that were directly extractable from measured coincidence counts, this experiment requires the reconstruction of the density matrices to obtain the concurrence values. In the experiment we tune the reflectivity of the TDC and hence vary the photon-photon interaction strength. Various four-photon quantum states are tomographically measured and the density matrices of them are reconstructed ${ }^{55,56}$. The concurrence of the two-photon subsystems is calculated from the four-photon density matrices by tracing out the other two photons.

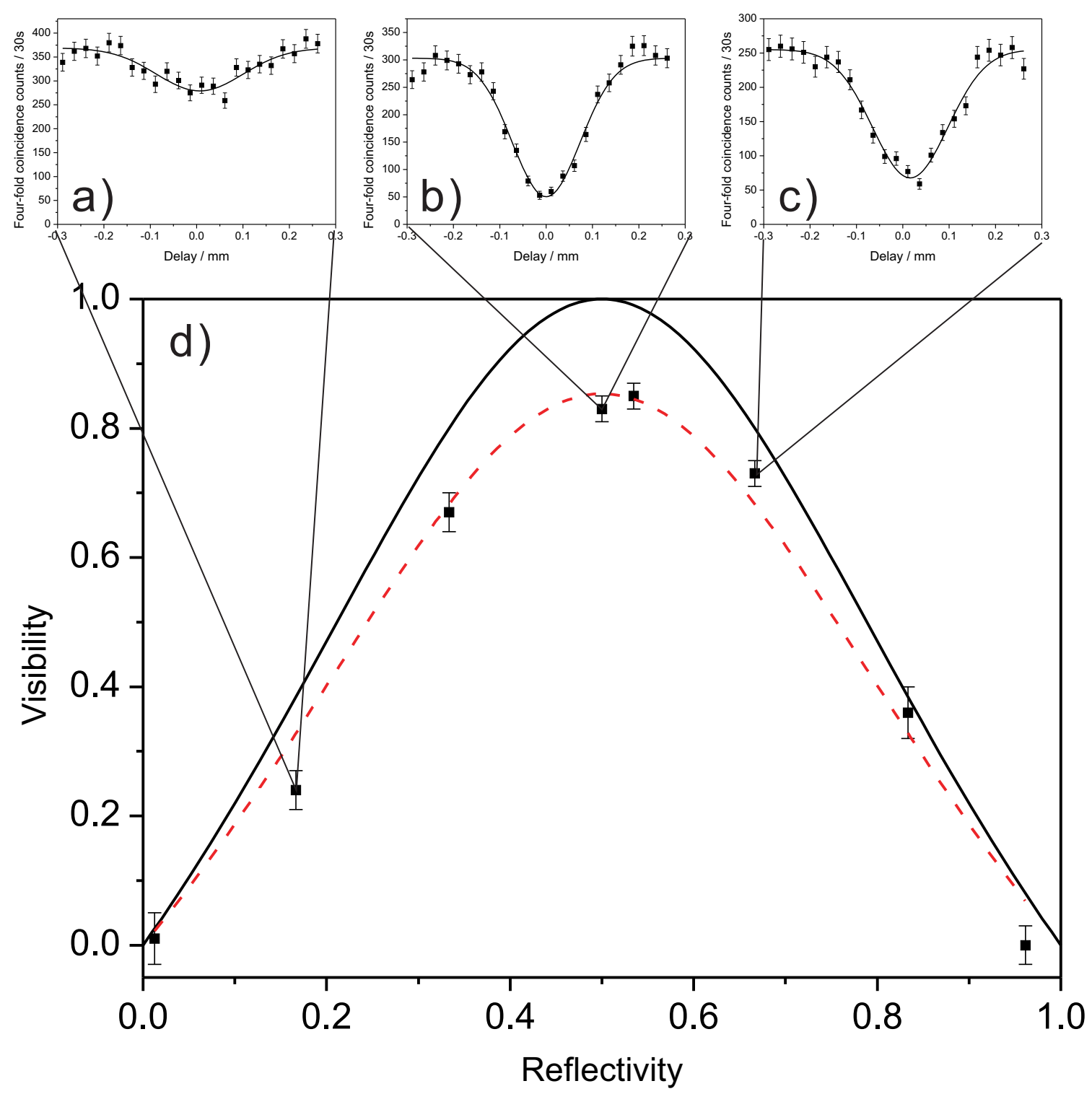

Figure 2 Experimental demonstration of variable measurement-induced interaction between two independent photons. (a), (b) and (c) Measured four-fold coincidence counts are plotted versus the relative optical delay between the interfering photons and fitted with a Gaussian function, when the reflectivities of the TDC are tuned to be $0.17,0.5$ and 0.67 , respectively. (d) Visibility of the Hong-Ou-Mandel (HOM) dip when measuring four-fold coincidences using a TDC. The black squares are the experimental results. The black solid curve is a theoretical prediction based on Eq. 1 and the red dashed curve is a fit with the only free parameter of $\mathrm{V}_{s y s}$, which is about 0.853 . The main reason of this non-ideal value is due to the higher-order emission generated from SPDC sources. The error bars are based on a Poissonian statistics. 


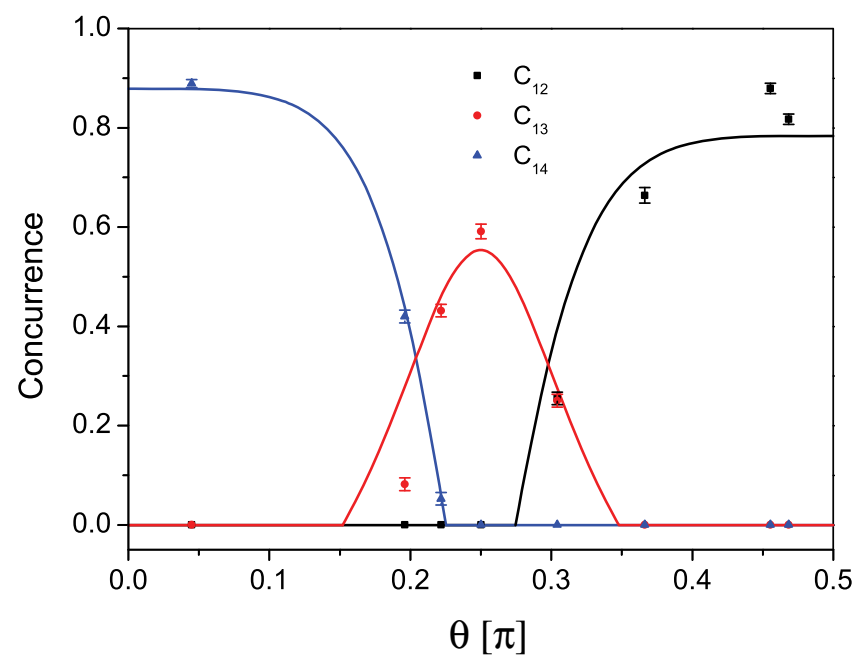

Figure $3 \mid$ Measured concurrence for various ground state configurations. Entanglement distribution between photons 1 \& 2 (black square, fitted with black curve), $1 \& 3$ (red circle, fitted with red curve) and $1 \& 4$ (blue triangle, fitted with blue curve) are shown as a function of the TDC parameter $\theta$. The rise of entanglement in one pair causes the fall of the entanglement in other pairs according to monogamy. The monogamy gives rise of the entanglement sudden death and sudden birth ${ }^{57-59}$. We derive the uncertainties in concurrence from the density matrices, which are calculated using a Monte Carlo routine and assumed Poissonian errors.

Due to the quantum monogamy relations $s^{31,46,47}$ the total amount of pair-wise entanglement stays constant while the change of interaction strength affects the distribution and thus the ground state configurations. When tracking the change in entanglement by using concurrence, the expected "sudden death" and "sudden birth" of entanglement ${ }^{58}$ can be seen (Fig. 3). While this concept is typically used for studying environment-induced decoherence ${ }^{59}$, a similar behavior can be observed here too. In fact our tunable interactions allow to mimic a controlled interaction with the environment of two additional particles, which opens the possibility to obtain insights into complex decoherence mechanisms. We explicitly show the concurrence of different photon-pair configuations with respect to the TDC angle $\theta$. The relation of the TDC angle and its reflectivity is given by $\theta=\arctan \sqrt{\eta}$. One can see that the concurrence for one photon pair (e.g. $C_{14}$ ) decreases rapidly as we increase $\theta$. At $\theta=$ 0.274 , all of a sudden the entanglement between another spin pair (e.g. $C_{13}$ ) is born at the cost of the reduced concurrence $C_{14}$, which vanishes as $\theta$ is further increased. The observation of similar disappearance or emergence of entanglement among the other photons demonstrates the capability of our quantum simulator for manipulating the quantum correlations ${ }^{57-59}$. a)

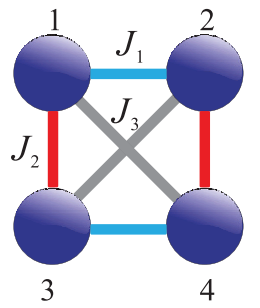

b)

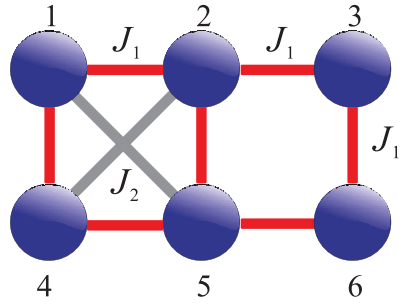

Figure $4 \mid$ (a) A four-site square lattice with $J_{1}$ being horizontal coupling, $J_{2}$ being vertical coupling and $J_{3}$ being diagonal coupling. (b) A six-site checkerboard lattice. $J_{1}$ is the coupling between the nearest neighbors and $J_{2}$ is the coupling on the cross bonds.

Generalized heisenberg spin model on a four-site square lattice and a six-site checkerboard lattice. For the four-site square lattice we extend the model in Ref. 31 by adding a next-nearest neighbor interaction term (Fig. 4a). The Hamiltonian for this system is:

$$
H=J_{1}\left(\mathbf{S}_{1} \cdot \mathbf{S}_{2}+\mathbf{S}_{3} \cdot \mathbf{S}_{4}\right)+J_{2}\left(\mathbf{S}_{1} \cdot \mathbf{S}_{3}+\mathbf{S}_{2} \cdot \mathbf{S}_{4}\right)+J_{3}\left(\mathbf{S}_{1} \cdot \mathbf{S}_{4}+\mathbf{S}_{2} \cdot \mathbf{S}_{3}\right)
$$

where $S_{i}$ is the Pauli spin operator for spin $i$ and $J_{1}, J_{2}$ and $J_{3}$ are the coupling strength parameters between different spins, respectively (see Fig. 4a).

We consider the antiferromagnetic case with the couplings $J_{1}, J_{2}$, $J_{3} \geq 0$. For each of the three terms in $H$, the ground state is a pair of singlets, $\left|\Phi_{=}\right\rangle=\left|\Psi_{12}^{-}\right\rangle\left|\Psi_{34}^{-}\right\rangle,\left|\Phi_{\|}\right\rangle=\left|\Psi_{13}^{-}\right\rangle\left|\Psi_{24}^{-}\right\rangle$and $\left|\Phi_{\times}\right\rangle=$ $\left|\Psi_{14}^{-}\right\rangle\left|\Psi_{23}^{-}\right\rangle=\left|\Phi_{=}\right\rangle-\left|\Phi_{\|}\right\rangle$(up to normalization). In Ref. 31 we showed that by tuning $J_{2} / J_{1}$ from 0 to $\infty$, the ground state, $\Phi_{g}$, gradually changes from $\left|\Phi_{=}\right\rangle$to $\left|\Phi_{\|}\right\rangle$.

With the introduction of $J_{3}$, the ground state of the system is still a superposition $\left|\Phi_{g}\right\rangle=\alpha\left|\Phi_{=}\right\rangle+\beta\left|\Phi_{\|}\right\rangle$with normalization condition $2\left(|\alpha|^{2}+|\beta|^{2}+|\alpha+\beta|^{2}\right)=1$.

Remarkably, tuning $J_{3} / J_{1}$ can induce sharp phase transitions with a sudden change of the ground state configuration due to the competing of the valence bond configurations. In Fig. 5, the ground state configurations for different coupling regimes are shown. There are three particular interesting phase transitions:

- For $J_{1}=J_{3},\left|\Phi_{g}\right\rangle$ suddenly changes from $\left|\Phi_{=}\right\rangle+\left|\Phi_{<}\right\rangle$to $\left|\Phi_{\|}\right\rangle$when $J_{2} / J_{1}$ is tuned across 1

- For $J_{2}=J_{3},\left|\Phi_{g}\right\rangle$ suddenly changes from $\left|\Phi_{=}\right\rangle$to $\left|\Phi_{\|}\right\rangle-\left|\Phi_{\times}\right\rangle$when $J_{2} / J_{1}$ is tuned across 1

- For $J_{1}=J_{2},\left|\Phi_{g}\right\rangle$ suddenly changes from $\left|\Phi_{=}\right\rangle+\left|\Phi_{\|}\right\rangle$to $\left|\Phi_{\times}\right\rangle$when $J_{3} / J_{1}$ is tuned across 1

The case with $J_{1}=J_{2} \neq J_{3}$ is widely studied for square lattice systems due to its relevance to cuprates, Fe-based superconductors, and other materials ${ }^{60-63}$. Previous studies have shown that in the thermodynamic limit, when $J_{3}>J_{1}$, the system is in a diagonal Neel ordered state ${ }^{64}$, which is consistent with the ground state $\left|\Phi_{\times}\right\rangle$
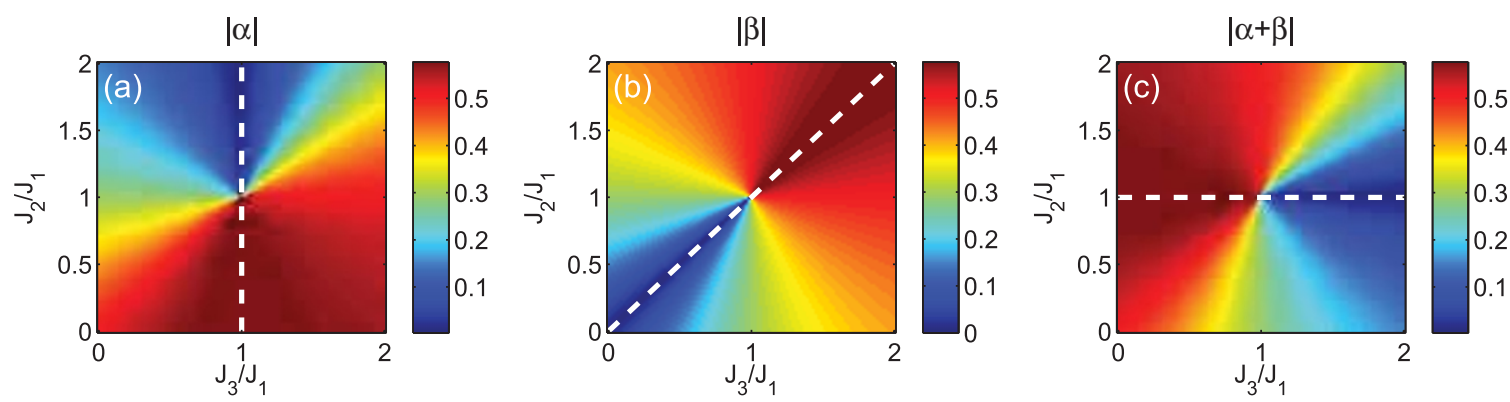

Figure $5 \mid$ Ground state phase diagram for various parameter regimes. The coefficients of the ground state, $\left|\Phi_{g}\right\rangle=\alpha\left|\Phi_{=}\right\rangle+\beta\left|\Phi_{\|}\right\rangle$, are shown for different ratios of $J_{2} / J_{1}$ and $J_{3} / J_{1}$. In $(\mathbf{a}),(\mathbf{b})$ and $(\mathbf{c})$, we show $|\alpha|,|\beta|$ and $|\alpha|+|\beta|$, respectively. A clear phase transition can be seen along the dashed line a) for $J_{1}=J_{3}, \mathbf{b}$ ) for $J_{2}=J_{3}$, and $\mathbf{c}$ ) for $J_{1}=J_{2}$. 


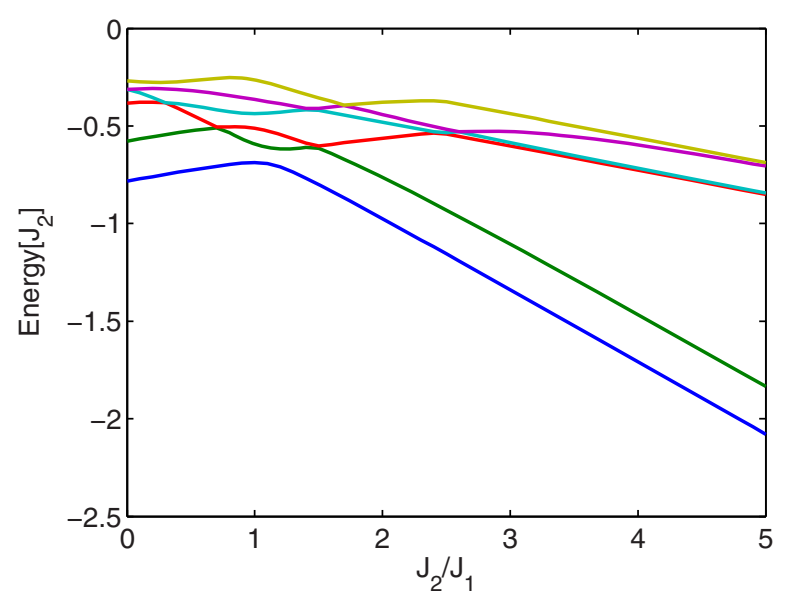

Figure 6 Energy spectrum of the low lying eigenstates of the six-site checkerboard lattice system. The energy levels are shown as a function of the ratio $J_{2} / J_{1}$. For the ratio $J_{2} / J_{1}=1$ an avoid level crossing of the ground state occurs.

for a minimum of four sites, as discussed above. In the regime where $J_{2}<J_{1}$ the configuration for the ground state is still under debate due to regions that appear to be non magnetic. Numerical calculation have recently shown that this region is highly likely to be a quantum spin liquid with $Z_{2}$ topological order ${ }^{64}$. However, for only four spins, the region with $J_{2}<J_{1}$ has only a single ground state configuration $\left|\Phi_{=}\right\rangle+\left|\Phi_{\|}\right\rangle$.

We also investigate theoretically the ground states of a $J_{1}-J_{2}$ Heisenberg model on a six-site checkerboard lattice. The geometry of this system is shown in Fig. $4 \mathrm{~b}$, where $J_{1}$ is the coupling strength between the nearest neighbor sites and $J_{2}$ is the coupling strength on the cross bonds. The coupling ratio $J_{2} / J_{1}$ is the only parameter of this system. The introduction of the next nearest neighbor coupling $J_{2}$ makes this system a simple frustrated magnetic model. The study of this model on thermodynamics limits is motivated by the threedimensional pyrochlore materials ${ }^{65,66}$. The two-dimensional model has been studied by several groups ${ }^{67-73}$. It is known that for the regime where the coupling ratio $J_{2} / J_{1} \ll 1$ the system has a co-linear Neel order. At $J_{2} / J_{1} \approx 1$ numerical calculation ${ }^{68}$ suggests a plaquette

a)
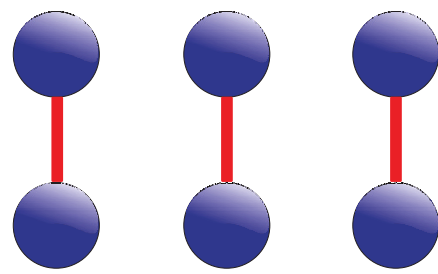

c)
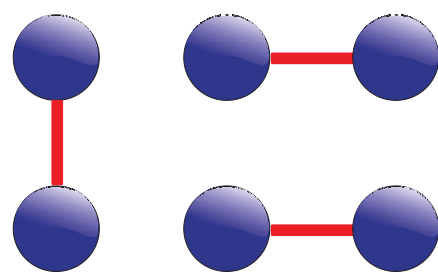

b)

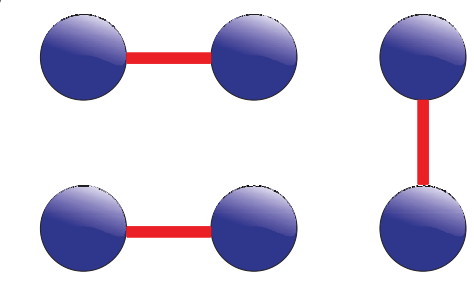

\section{.}

d)

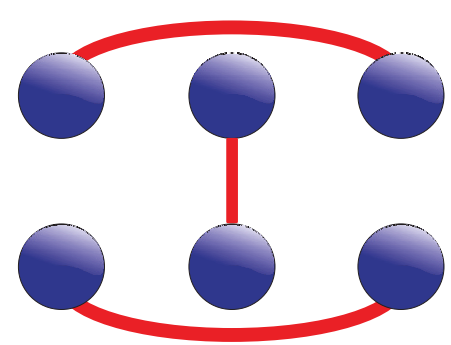

valence bond solid ground state while the ground state at $J_{2} / J_{1} \gg 1$ remains under debate ${ }^{67,69,70}$. For this reason future photonic quantum simulations might provide answers to these open questions.

Numerical studies of the six-site checkerboard system were done by diagonalizing the Hamiltonian with open boundary conditions. In Fig. 6 the energy spectrum of the six low lying states as a function of the ratio $J_{2} / J_{1}$ in the $\sum_{i} S_{i}^{z}=0$ subspace is presented. The energy spectrum shows an avoid level crossing around $J_{2} / J_{1} \approx 1$, which indicates a dramatic change of the ground state properties there. In analogy to the $J_{1}-J_{2}$ Heisenberg model on a plaquette, where the ground states can be expressed as linear superposition of two different dimer coverings whose coefficients depend on the ratio $J_{2} / J_{1}$, the ground states for the six-site checkerboard lattice can also be expressed as superpositions of various dimer configurations. For the discussed six-site lattice system, fifteen different dimer coverings exist. However, only six are independent. By taking the symmetry of our system into consideration only four out of the six coverings are allowed (Fig. 7).

Therefore, the ground states can be described as superpositions of four dimer coverings, $\left|\psi_{1}\right\rangle,\left|\psi_{2}\right\rangle,\left|\psi_{3}\right\rangle,\left|\psi_{4}\right\rangle$ in all region of $J_{2} / J_{1}$. In Fig. 8 we show the contribution of each dimer configuration with respect to the coupling ratio $J_{2} / J_{1}$. At $J_{2} / J_{1}=1$ the coefficients for $\left|\psi_{1}\right\rangle$ and $\left|\psi_{3}\right\rangle$ are equal and coefficients for $\left|\psi_{2}\right\rangle$ and $\left|\psi_{4}\right\rangle$ are exact zero. In our current convention of the dimer covering wave function this particular superposition gives us a plaquette state on the right four sites as it is shown in the inset. This coincides with the plaquette valence bond solid state in an infinite system. Remarkably, the sixsite checkerboard lattice thus provides already a valuable hint for the true ground state in the thermodynamic limit. The ground states at the ratio $J_{2}>J_{1}$ have close to equal contribution from $\left|\psi_{1}\right\rangle$ and $\left|\psi_{2}\right\rangle$, which suggests a significant contribution from a cross-dimer state on the left plaquette. We would like to mention that a cross-dimer ground state has also been suggested as a potential ground state for large $J_{2}$ couplings $^{69}$. It is interesting that only a small contribution from the dimer configuration $\left|\psi_{4}\right\rangle$ can be found in all the possible cases.

Summary and outlook. In conclusion, today's available photonic quantum technology is reaching the stage where significant advantages arise for the simulation of particular interesting questions in solid-state physics and quantum chemistry. Therefore photonic

Figure $7 \mid$ The independent ground state configurations of the six-site checkerboard lattice. The four different dimer coverings are labeled as (a) $\left|\psi_{1}\right\rangle$, (b) $\left|\psi_{2}\right\rangle,(\mathrm{c})\left|\psi_{3}\right\rangle$, and (d) $\left|\psi_{4}\right\rangle$ in our system. 


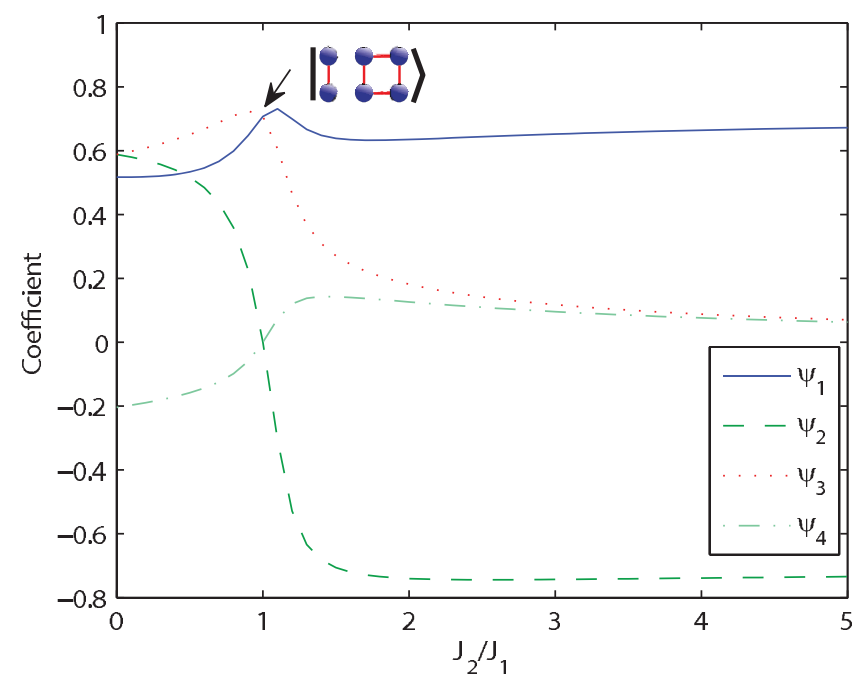

Figure 8 The six-site checkerboard lattice ground state. The according superposition of the four independent dimer configurations, $\left|\psi_{1}\right\rangle,\left|\psi_{2}\right\rangle$, $\left|\psi_{3}\right\rangle,\left|\psi_{4}\right\rangle$ is shown as function of the ratio $J_{2} / J_{1}$. The inset shows the plaquette ground state at $J_{2} / J_{1}=1$.

quantum simulations provide exciting opportunities to cover, for example, the direct construction of custom-tailored many-body wave functions. Impressively, the usage of optical elements such as tunable non-polarizing or polarizing beam splitters enables entangled few-photon states to construct many-body valence bond wave functions for molecular and solid-state systems due to nonclassical interferences. As we have shown above, this is of particular interest in condensed matter physics as it provides insight into the frustration of strongly-correlated spin systems and the onset of quantum phase transitions. On the other hand, in quantum chemistry it effectively allows studying delocalized bonds in chemical structures and chemical reactions ${ }^{6}$. Thus being able to monitor the full dynamics of individual particles and bonds provide some fascinating perspective for the quantum simulation of small molecules or reactive centers.

The main future challenge will be to increase the number of photons or degrees of freedom to realize a sufficient amount of qubits such that quantum computers can outperform their classical counter parts. In general, up to the level of approximately twenty qubits it presently appears possible to conceive a system based on bare physical qubits. However, given the current experimental limitations, operational fidelities and noise sources, it seems that useful system consisting of more than twenty qubits could not be realized without some level of error correction. But in contrast to the implementations of well-known quantum algorithms, such as Shor's algorithm for a computationally relevant key-length, the requirements for faulttolerance are much less demanding. As such an open problem relates to how much error correction is needed to achieve a useful quantum simulation.

Recent work ${ }^{6,27}$ has shown that quantum systems with less than a dozen physical qubits are capable of simulating chemical systems with a precision that cannot be achieved by conventional computers, when processed via almost a thousand discrete gate operations. Although such small quantum systems are feasible by using present quantum technology, the requirements in terms of gate operations is tremendous. Thus, analog quantum gate operations look promising in reducing the technical complexity of performing such quantum simulation experiments by requiring fewer number of physical gates.

1. Feynman, R. Simulating physics with computers. Int. J. Theor. Phys. 21, 467-488 (1982).
2. Feynman, R. P. Quantum mechanical computers. Found. Phys. 16, 507-531 (1986).

3. Lloyd, S. Universal quantum simulators. Science 273, 1073-1078 (1996).

4. Buluta, I. \& Nori, F. Quantum simulators. Science 326, 108-111 (2009).

5. Georgescu, I. M., Ashhab, S. \& Nori, F. Quantum Simulation. arXiv:quant-ph/ 1308.6253 (2013).

6. Aspuru-Guzik, A., Dutoi, A. D., Love, P. J. \& Head-Gordon, M. Simulated quantum computation of molecular energies. Science 309, 1704-1707 (2005).

7. Trebst, S., Schollwöck, U., Troyer, M. \& Zoller, P. d-wave resonating valence bond states of fermionic atoms in optical lattices. Phys. Rev. Lett. 96, 250402 (2006).

8. Kassal, I., Jordan, S. P., Love, P. J., Mohseni, M. \& Aspuru-Guzik, A. Polynomialtime quantum algorithm for the simulation of chemical dynamics. PNAS 105, 18681-18686 (2008).

9. Verstraete, F., Cirac, J. I. \& Latorre, J. I. Quantum circuits for strongly correlated quantum systems. Phys. Rev. A 79, 032316 (2009).

10. Lewenstein, M. et al. Ultracold atomic gases in optical lattices: mimicking condensed matter physics and beyond. Adv. Phys. 56, 243-379 (2007).

11. Bakr, W. S., Gillen, J. I., Peng, A., Folling, S. \& Greiner, M. A quantum gas microscope for detecting single atoms in a hubbard-regime optical lattice. Nature 462, 74-77 (2009).

12. Bakr, W. S. et al. Probing the superfluid-to-mott insulator transition at the singleatom level. Science 329, 547-550 (2010).

13. Trotzky, S. et al. Suppression of the critical temperature for superfluidity near the Mott transition. Nat. Phys. 6, 988-1004 (2010).

14. Sherson, J. F. et al. Single-Atom Resolved Fluorescence Imaging of an Atomic Mott Insulator. Nature 467, 68-72 (2010)

15. Weitenberg, C. et al. Single-spin addressing in an atomic Mott insulator. Nature 471, 319-324 (2011).

16. Friedenauer, A., Schmitz, H., Glueckert, J. T., Porras, D. \& Schaetz, T. Simulating a quantum magnet with trapped ions. Nat. Phys. 4, 757-761 (2008).

17. Gerritsma, R. et al. Quantum simulation of the dirac equation. Nature 463, 68-71 (2010).

18. Kim, K. et al. Quantum simulation of frustrated ising spins with trapped ions. Nature 465, 590-593 (2010).

19. Barreiro, J. T. et al. An open-system quantum simulator with trapped ions. Nature 470, 486-491 (2011).

20. Islam, R. et al. Onset of a quantum phase transition with a trapped ion quantum simulator. Nat. Comm. 2, 377 (2011).

21. Lanyon, B. P. et al. Universal Digital Quantum Simulation with Trapped Ions. Science 334, 57-61 (2011).

22. Peng, X., Zhang, J., Du, J. \& Suter, D. Quantum simulation of a system with competing two- and three-body interactions. Phys. Rev. Lett. 103, 140501 (2009).

23. Du, J. et al. NMR implementation of a molecular hydrogen quantum simulation with adiabatic state preparation. Phys. Rev. Lett. 104, 030502 (2010).

24. Neeley, M. et al. Emulation of a Quantum Spin with a Superconducting Phase Qudit. Science 325, 722-725 (2009).

25. Lu, C.-Y. et al. Demonstrating anyonic fractional statistics with a six-qubit quantum simulator. Phys. Rev. Lett. 102, 030502 (2009).

26. Pachos, J. K. et al. Revealing anyonic features in a toric code quantum simulation. New J. Phys. 11, 083010 (2009).

27. Lanyon, B. P. et al. Towards quantum chemistry on a quantum computer. Nat. Chem. 2, 106-111 (2010).

28. Broome, M. A. et al. Discrete Single-Photon Quantum Walks with Tunable Decoherence. Phys. Rev. Lett. 104, 153602 (2010).

29. Peruzzo, A. et al. Quantum Walks of Correlated Photons. Science 329, 1500-1503 (2010).

30. Kaltenbaek, R., Lavoie, J., Zeng, B., Bartlett, S. D. \& Resch, K. J. Optical one-way quantum computing with a simulated valence-bond solid. Nat. Phys. 6, 850-854 (2010).

31. Ma, X.-s, Dakić, B., Naylor, W., Zeilinger, A. \& Walther, P. Quantum simulation of the wavefunction to probe frustrated Heisenberg spin systems. Nat. Phys. 7, 399-405 (2011)

32. Matthews, J. C. F. et al. Simulating quantum statistics with entangled photons: a continuous transition from bosons to fermions. arXiv:quant-ph/1106.1166 (2011).

33. Sansoni, L. et al. Two-Particle Bosonic-Fermionic Quantum Walk via Integrated Photonics. Phys. Rev. Lett. 108, 010502 (2012).

34. Bloch, I., Dalibard, J. \& Nascimbéne, S. Quantum simulations with ultracold quantum gases. Nat. Phys. 8, 267-276 (2012).

35. Blatt, R. \& Roos, C. F. Quantum simulations with trapped ions. Nat. Phys. 8, 277-284 (2012).

36. Aspuru-Guzik, A. \& Walther, P. Photonic quantum simulators. Nat. Phys. 8, 285-291 (2012).

37. Houck, A. A., Türeci, H. E. \& Koch, J. On-chip quantum simulation with superconducting circuits. Nat. Phys. 8, 292-299 (2012).

38. Knill, E., Laflamme, R. \& Milburn, G. A scheme for efficient quantum computation with linear optics. Nature 409, 46-52 (2001).

39. Langford, N. K. et al. Efficient quantum computing using coherent photon conversion. Nature 478, 360 (2011).

40. Mattle, K., Weinfurter, H., Kwiat, P. G. \& Zeilinger, A. Dense Coding in Experimental Quantum Communication. Phys. Rev. Lett. 76, 4656-4659 (1996). 
41. Anderson, P. W. The resonating valence bond state in $\mathrm{La}_{2} \mathrm{CuO}_{4}$ and superconductivity. Science 235, 1196-1198 (1987).

42. Marshall, W. Antiferromagnetism. Proc. R. Soc. A 232, 48-68 (1955).

43. Lieb, E. \& Mattis, D. Ordering energy levels of interacting spin systems. J. Math. Phys. 3, 749-751 (1962).

44. Affleck, I., Kennedy, T., Lieb, E. H. \& Tasaki, H. Rigorous results on valence-bond ground states in antiferromagnets. Phys. Rev. Lett. 59, 799-802 (1987).

45. Balents, L. Spin liquids in frustrated magnets. Nature 464, 199-208 (2010).

46. Coffman, V., Kundu, J. \& Wootters, W. K. Distributed entanglement. Phys. Rev. A 61, 052306 (2000).

47. Osborne, T. J. \& Verstraete, F. General monogamy inequality for bipartite qubit entanglement. Phys. Rev. Lett. 96, 220503 (2006).

48. Kwiat, P. G. et al. New high-intensity source of polarization-entangled photon pairs. Phys. Rev. Lett. 75, 4337-4341 (1995).

49. Hong, C. K., Ou, Z. Y. \& Mandel, L. Measurement of subpicosecond time intervals between two photons by interference. Phys. Rev. Lett. 59, 2044-2046 (1987).

50. Kok, P. et al. Linear optical quantum computing with photonic qubits. Rev. Mod. Phys. 79, 135-174 (2007).

51. Pan, J. W., Chen, Z. B., Żukowski, M., Weinfurter, H. \& Zeilinger, A. Multi-photon entanglement and interferometry. Rev. Mod. Phys. 84, 777-838 (2012).

52. Pan, J. W., Bouwmeester, D., Weinfurter, H. \& Zeilinger, A. Experimental Entanglement Swapping: Entangling Photons That Never Interacted. Phys. Rev. Lett. 80, 3891-3894 (1998).

53. Jennewein, T., Weihs, G., Pan, J. W. \& Zeilinger, A. Experimental Nonlocality Proof of Quantum Teleportation and Entanglement Swapping. Phys. Rev. Lett. 88, 017903 (2002).

54. Wootters, W. K. Entanglement of formation of an arbitrary state of two qubits. Phys. Rev. Lett. 80, 2245-2248 (1998).

55. White, A. G., James, D. F. V., Eberhard, P. H. \& Kwiat, P. G. Nonmaximally entangled states: Production, characterization, and utilization. Phys. Rev. Lett. 83, 3103-3107 (1999).

56. James, D. F. V., Kwiat, P. G., Munro, W. J. \& White, A. G. Measurement of qubits. Phys. Rev. A 64, 052312 (2001).

57. Almeida, M. P. et al. Environment-Induced Sudden Death of Entanglement. Science 316, 579-582 (2007).

58. Yu, T. \& Eberly, J. H. Sudden Death of Entanglement. Science 323, 598-601 (2009).

59. Jimenez Farias, O., Lombard Latune, C., Walborn, S. P., Davidovich, L. \& Souto Ribeiro, P. H. Determining the Dynamics of Entanglement. Science 324, 1414-1417 (2009)

60. Dagotto, E. \& Moreo, A. Phase diagram of the frustrated spin-1/2 Heisenberg antiferromagnet in 2 dimensions. Phys. Rev. Lett. 63, 2148-2151 (1989).

61. Melzi, R. et al. Magnetic and thermodynamic properties of $\mathrm{Li}_{2} \mathrm{VOSiO}_{4}$ : A twodimensional $S=1 / 2$ frustrated antiferromagnet on a square lattice. Phys. Rev. $B$ 64, 024409 (2001).

62. Fang, C. et al. Theory of electron nematic order in LaFeAsO. Phys. Rev. B 77, 224509 (2008)

63. Mezzacapo, F. Ground-state phase diagram of the quantum $\mathrm{J}_{1}-\mathrm{J}_{2}$ model on the square lattice. Phys. Rev. B 86, 045115 (2012).

64. Jiang, H.-C., Yao, H. \& Balents, L. Spin liquid ground state of the spin- $1 / 2$ square $\mathrm{J}_{1}$ - $\mathrm{J}_{2}$ Heisenberg model, textitPhys. Rev. B 86, 024424 (2012).

65. Axtell III, E. A., Ozawa, T., Kauzlarich, S. M. \& Singh, R. R. P. Phase Transition and Spin-gap Behavior in a Layered Tetragonal Pnictide Oxide. J. Solid State Chem. 134, 423-426 (1997).

66. Coldea, R., Tennant, D. A., Tsvelik, A. M. \& Tylczynski, Z. Experimental Realization of a 2D Fractional Quantum Spin Liquid. Phys. Rev. Lett. 86, 1335-1338 (2001).
67. Sindzingre, P., Fouet, J. B. \& Lhuillier, C. One-dimensional behavior and sliding Luttinger liquid phase in a frustrated spin-1/2 crossed chain model: Contribution of exact diagonalizations. Phys. Rev. B 66, 174424 (2002).

68. Starykh, O. A., Singh, R. R. P. \& Levine, G. C. Spinons in a Crossed-Chains Model of a 2D Spin Liquid. Phys. Rev. Lett. 88, 167203 (2002).

69. Staryhk, O. A., Furusaki, A. \& Balents, L. Anisotropic pyrochlores and the global phase diagram of the checkerboard antiferromagnet. Phys. Rev. B 72, 094416 (2005).

70. Moukouri, S. Néel and valence-bond crystal phases of frustrated two-dimensional Heisenberg models. Phys. Rev. B 77, 052408 (2008).

71. Brenig, W. \& Grzeschik, M. Valence-bond crystal phase of the crossed-chain quantum spin model. Phys. Rev. B 69, 064420 (2004).

72. Tchernyshyov, O., Starykh, O. A., Moessner, R. \& Abanov, A. G. Bond order from disorder in the planar pyrochlore magnet. Phys. Rev. B 68,144422 (2003).

73. Bernier, J. S., Chung, C., Kim, Y. \& Sachdev, S. Planar pyrochlore antiferromagnet: A large-N analysis. Phys. Rev. B 69, 214427 (2004).

\section{Acknowledgments}

X.S.M., B.D., S.K., W.N., Y.H.C., A.Z. and P.W. thank F. Verstraete and Č. Brukner for helpful discussions. We further acknowledge support from the European Commission, Q-ESSENCE (No. 248095), ERC Advanced Senior Grant (QIT4QAD), QUILMI (No. 295293), EQUAM (No. 323714), PICQUE (No. 608062), GRASP (No. 613024) and the ERA-Net CHISTERA project QUASAR, the John Templeton Foundation, the Vienna Center for Quantum Science and Technology (VCQ), the Austrian Nanoinitiative NAP Platon, the Austrian Science Fund (FWF) through the SFB FoQuS (No. F4006-N16), START (No. Y585-N20) and the doctoral programme CoQuS, the Vienna Science and Technology Fund (WWTF) under grant ICT12-041, and the Air Force Office of Scientific Research, Air Force Material Command, United States Air Force, under grant number FA8655-11-1-3004. XSM was supported by a Marie Curie International Outgoing Fellowship within the $7^{\text {th }}$ European Community Framework Programme. Y.H.C., Z.X.G. and L.M.D. thank H.-C. Jiang for helpful discussion and acknowledge support from the NBRPC (973 Program) 2011CBA00300 (2011CBA00302) and the DARPA OLE program.

\section{Author contributions}

X.S.M., S.K. and W.N. designed and performed experiments, analyzed data and wrote the manuscript. B.D. provided the theoretical analysis, analyzed data and wrote the manuscript. Y.H.C., Z.X.G. and L.M.D. developed the theoretical analysis of the general Heisenberg spin model on 4-site and 6-site lattices. A.Z. supervised the project and edited the manuscript. P.W. designed experiments, analyzed data, wrote the manuscript and supervised the project.

\section{Additional information}

Competing financial interests: The authors declare no competing financial interests.

How to cite this article: Ma, X.-s. et al. Towards photonic quantum simulation of ground states of frustrated Heisenberg spin systems. Sci. Rep. 4, 3583; DOI:10.1038/srep03583 (2014).

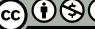

This work is licensed under a Creative Commons AttributionNonCommercial-NoDerivs 3.0 Unported license. To view a copy of this license, visit http://creativecommons.org/licenses/by-nc-nd/3.0 